\title{
Prevalencia de anemia ferropriva y deficiencia de hierro en una población materno infantil del Area Sur Oriente de Santiago de Chile. 1970
}

\author{
DRES. AUGUSTO WINTER *, HERNAN TABOADA *, ALBERTO GALOFRE *, ALBERTO MAIZ * y AN"- \\ TONIO ARTEAGA *
}

InTroducclón. La deficiencia de hierro constituye uno de los principales problemas nutricionales y es la primera causa de anemia en la población mundial (1). Su reconocimiento precoz y tratamiento, lleva a una rápida y franca mejoría de la salud y de la capacidad física e intelectual de los afectados, siendo ello posible a travês de programas de relativo bajo costo. (2) (3).

Se calcula que en América del Sur, la prevalencia de ancmia ferropriva oscila entre un 5 y un $15 \%$ para los bombres y en un 10 a $35 \%$ para las mujeres. El porcentaje de niños anémicos varía entre 15 y $50 \%$ (1).

Los grupos más expuestos parecen ser los niños entre 6 y 18 meses y las mujeres gestantes.

La metodología usual para determinat la prevalencia de la deficiencia de hierro, ha sido la medición de la hemoglobina de la sangre, el hemaw tocrito y el valor derivado: concentración hemoglobínica corpuscular media. Estos procedimientos reflejan la etapa final del proceso y sólo una parte del problema.

Al utilizar el porcentaje de saturación de la transferrina como criterio de diagnóstico de la deficiencia de hierro, se obtiene una visión real deI trastorno que tratamos. Así, se acepta como deficiente una saturación menor del $15 \%$, el rango de la prevalencia de la deficiencia de hierro en las gestantes latinoamericanas, sube del $20-80 \%$ al $40-99 \%$ (1).

En Chile existen algunos estudios (4) (5) (6) (7) (8) (9) que señalan la prevalencia de la anemia ferropriva y sólo en uno de ellos se destaca la deficiencia de hierro, señalando que el $62,5 \%$ de los lactantes eutróficos del Area Norte

* Facultad de Medicina. Universidad Catóica. Departamento de Pediatría y Sección de Nutríción del Departamenio de Medicina. Hospilal Sotero del Río. de Santiago de Chile, presentan una saturación de la transfcrrina inferior al $15 \%$ (5).

Por lo expresado anteriormente, hemos creído de interés estudiar la prevalencia de la deficiencia de hierro en una población materno-infantil de un área representativa de la población proletaria del gran Santiago. Igualmente deseamos objetivar la validez de los distintos indicadores utilizados co. múnmente en el diagnóstico de dicho problema.

Señalamos también que en otro trabajo (10), presentamos el papel de la dieta en la patogenia de la deficiencia de hierro en la nisma pcb'ación, a igual que al impacto de esta patología sobre el estuło nutritivo de la población infantil.

Métodos. Este estudio se realizó en la comuna de La Granja, ubicada en el sector Sur Oricnte del gran Santiago, con una pob'ación que bordea los 200.000 habitantes. Corresponde al Area hos. pit:a'aria de Puente Alto, cuyo hospital base es el Sótero del Rio.

Se seleccionaron para la investigación, las pob.aciones beneficiarias de los consultorics San Gregorio (42.000 habts.), San Rafael (50.000 habts.) y La Granja ( 100,000 habts.).

El estudio se inició con la pesquisa de 600 recién nacidos vivos, 200 de cada consultorio $y$ cuyes partos se produjercn en el hospital base. Ello corresponde al $20 \%$ de los nacinientos estimados al año.

El prime: contacto se realizó entre las $12 y$ 24 horas de vida del recién nacido. El segundo, base de este estudio, a los 5 meses, en donde se examinó al lactante índice, a sus madres y a todos los niños menores de 14 añcs de las familias.

Este segundo control se intentó en la totalida t de la población definida, pero existió un porcen- 
taje de pérdida variable, por rechazo, migración o fallas técnicas en la extracción de sangre.

La muestra se obtuvo por punción venosa y en condiciones de ayunas. De inmediato se determinaron las concentraciones de hemoglobina de la sangre y el hematocrito, para luego guardar el suero a $-4 \% \mathrm{C}$, y proceder a las determinaciones de hicrro sérico y transferrina.

Para clasificar la población por grupos fisiológicos, se siguieron les siguientes criterios: lactantes: de 0 a 24 meses; preescolares: de 25 a 72 meses y escolares: de 73 a 180 meses. Se consideró como mujer nodriza a aquélla que lactaba en forma continuada a la fecha del estudio y como mujer no nodriza, a aquélla que no lactaba a su hijo. En ambos casos se descartó el embarazo por interrogatorio, dejando constancia de que se encontraban a $5-7$ meses de su parto anterior y no estaban bajo terapia anticonceptiva ni hierroterapia.

Como patrones de referencia se utilizaron los recomendados por organismos internacionales, los cuales se exponen en la tabla No 1. (11).

En Ja tabla No 2 se presenta la población estudiada, agrupada en conjuntos fisiológicos, junto a sus respectivas edades promedio. Se totalizaron 1.172 individuos: 404 lactantes $(34,4 \%)$, con edad promedio de 8 meses y con un rango entre 5 y 24 meses; 217 preescolares $(18,5 \%) ; 226$ escolares $(19,2 \%) ; 189$ mujeres no nodrizas $(16,1 \%)$ y 136 mujeres nodrizas $(11,8 \%)$.

La hemoglobina se determinó según técnica de Cianmetahemoglobina (12); al hematocrito, según microhematocrito (13); el hierro sérico y la transferrina, según técnica de Bothwell y Mallett (14) (15), modificada por el Comité Internacional para Standarización en Hematología.

T A B L A Nơ 1

DEFINICION DE RANGOS DE REFERENCIA PARA LA POBLACION MATERNO INFANTIL ESTUDIADA

\begin{tabular}{l|c|c|c}
\hline & Deficiente & Bajo & $\begin{array}{c}\text { Probabie } \\
\text { Normal }\end{array}$ \\
\hline Población infantil & & & \\
Hematocrito $\%$ & $\leqslant 29$ & $30-33$ & $\geqslant 34$ \\
Hemoglobinemia gr\% & $\leqslant 9$ & 10 & $\geqslant 11$ \\
C. H. C. M. \% & $\leqslant 27$ & $28-30$ & $\geqslant 31$ \\
Hierro sérico ugr\% & $\leqslant 30$ & $31-49$ & $\geqslant 50$ \\
$\%$ Sat. transferrina & $\leqslant 14$ & $15-19$ & $\geqslant 20$ \\
Población materra & & & \\
Hematocrito \% & $\leqslant 29$ & $30-37$ & $\geqslant 38$ \\
Hemoglobinemia gr\% & $\leqslant 9$ & $10-11$ & $\geqslant 12$ \\
C. H. C. M. \% & $\leqslant 27$ & $28-30$ & $\geqslant 31$ \\
Hierro sérico ugr $\%$ & $\leqslant 29$ & $30-49$ & $\geqslant 50$ \\
$\%$ Sat. transferrina & $\leqslant 14$ & $15-19$ & $\geqslant 20$ \\
\hline
\end{tabular}

TA B LA No 2

POBLACION ESTUDIADA. AREA SUR ORIENTE DE SANTIAGO. 1970

\begin{tabular}{l|c|c|c}
\hline Grupo & $\begin{array}{c}\text { Evad } \\
\text { promedio } \\
\text { (meses) }\end{array}$ & No casos & $\%$ \\
\hline Lactantes & 8 & 404 & $\mathbf{3 4 , 4}$ \\
Preescolares & 49 & 217 & 18,5 \\
Escolares & 113 & $\mathbf{2 2 6}$ & 19,2 \\
Madres no nodrizas & 299 & 189 & 16,1 \\
Madres nodrizas & 296 & 136 & 11,8 \\
\hline
\end{tabular}

\section{TABLA No 3}

VALORES YROMEDIO DE HEMATOCRITO, HEMOGLOBINEMIA, CONCENTRACION HEMOGLOBINICA CORPUSCULAR MEDIA (C.H.C.M.), HIERRO SERICO, TRANSFERRINA $Y \%$ DE SATURACION DE ELLA, EN UNA POBLACION INFANTIL DEL AREA SUR ORIENTE DE SANTIAGO. 1970

\begin{tabular}{|c|c|c|c|c|c|c|}
\hline \multirow{2}{*}{ INDICADOR } & \multicolumn{2}{|c|}{$\begin{array}{c}\text { Lactantes } \\
404\end{array}$} & \multicolumn{2}{|c|}{$\begin{array}{c}\text { Preescolares } \\
2 \sqrt{17}\end{array}$} & \multicolumn{2}{|c|}{$\begin{array}{c}\text { Escolares } \\
226\end{array}$} \\
\hline & $x$ & D.S. & $\vec{x}$ & $D . S$ & $\bar{x}$ & D.S. \\
\hline Hematocrito sh & 33,4 & 3,6 & 35,8 & 3,8 & 3.8 .2 & 3,1 \\
\hline Hernoglobinem'i $\mathrm{gr}^{3 /}$ & 11,4 & 1,4 & 12,3 & 1.3 & 13,0 & 1.2 \\
\hline c. H. C. A. \% & 33,9 & 3,1 & 34,0 & 2,2 & 33.7 & 1,7 \\
\hline Hierro serico uge\% & 50,2 & 26,9 & 76,1 & 27.1 & 82,9 & 27,9 \\
\hline Transferrina $u g \%$ & 353.7 & 67,9 & 327,2 & 57.7 & 328,7 & 49,0 \\
\hline 9 Sat. transfer xina & 14.3 & 7,5 & 23,8 & 9,3 & 25,3 & 9,1 \\
\hline
\end{tabular}

TABLA No 4

VALORES PROMEDIO DE HEMATOCRITO, HEMO. GLOBINEMIA, CONCENTRACION HEMOGLOBANICA CORPUSCULAR MEDIA (C.H.C.M.). HIERRO SERICO, TRANSFERRINA Y \% DE SATURACJON DE ELLA, EN UNA POBLACION MATERNA DEL AREA SUR ORIENTE DE SANTIAGO. 1970

\begin{tabular}{|c|c|c|c|c|}
\hline \multirow[t]{2}{*}{ INDICADOR } & \multicolumn{2}{|c|}{$\begin{array}{c}\text { Mujer no } \\
\text { nodriza } 189\end{array}$} & \multicolumn{2}{|c|}{$\begin{array}{c}\text { Mujer nodriza } \\
\text { 1j6 }\end{array}$} \\
\hline & $\bar{x}$ & D.S. & $x$ & $D . S$ \\
\hline Hematocrito $\%$ & 38.7 & 3,5 & 37,9 & 3,9 \\
\hline Hemoglobitumsia gr\% & 12,9 & 1,2 & 12,9 & 1,6 \\
\hline C. H. C. M. \% & 33,4 & 1,6 & 33,9 & 2,5 \\
\hline Hierro sérico ugr\% & 74,1 & 33,1 & 81,6 & 34.5 \\
\hline Transferrina ugr $\%$ & 374,2 & 72,0 & 365,3 & 62,4 \\
\hline$\%$ Sat. transferrina & 20,0 & 8,6 & 22,4 & 10,3 \\
\hline
\end{tabular}

La concentración hemoglobínica corpuscular media se dcrivó de los valores de hematocrito y concentración de hemoglobina sérica, de acuerdo 


\begin{abstract}
$\mathrm{Hbgr} \% \times 100$
a la siguiente fórmula: $\frac{}{\text { Hcto } \%}=\%$.

El análisis de los resultados se realizó por computación, utilizando un computador Bourroghs 3.500 y con la asesoría tícnica de la Escuela de Ingeniería de la Universidod Católica.
\end{abstract}

Resultados. En las tablas № 3 y 4 se aprecian los valores promedio de los indicadores cstudiados en la población infantil y materna. Destaca que, con excepción de la población lactante, el resto de los valores promedio se encuentra dentro del rango de la normalidad.

En efecto, en los lactantes, el nivel de hemoglobinemia promedio se halla en cl rango "bajo" y el porcentaje de la saturación de la transferrina, en el "deficiente".

Otro hecho que se observa es el incremento de los valores promedio de hematocrito, hemoglobinemia, hierro sérico y \% de saturación de la transferrina. Este aumento progresivo en los indices analizados, desde la lactancia a la edad escolar, alcanza significación estadística $(P>0,01 \%)$. $\mathrm{La}$ variación es más notoria entre la lactancia y la edad preescolar.

Sin embargo, este carácter no se observa en la concentración hemoglobinica corpuscular media, que mantiene valores estables, ni en la transferrina, que presenta una significativa reducción $(\mathrm{P}>$ $0,01 \%$ ) entre la lactancia y las edades siguientes.

También existe una notable similitud de los valores de hematocrito, hemoglobinemia, concentración hemoglobínica corpuscular media y hierro sérico, entre la edad escolar y las madres. Hacen excepción, los valores de transferrina, que son significativamente más altos $(\mathbf{P}>0,01 \%), y$ los de saturación de ella, que son significativamente menores $(\mathrm{P}>0,01 \%)$ en estas últimas.

En la tabla No 4 no se aprecian diferencias significativas entre las mujeres nodrizas y no nodrizas.

En los gráficos N.os 1 al 6 se presentan los valores promedio de dichos indicadores por cdad y sexo. Se observa que los valores de hematocrito, hemoglobinemia, hierro sérico y $\%$ de saturación de la transferrina, incrementan con la edad, lo que es especialmente notorio entre los 5 y 30 meses de edad. Analizando los valores por sexo, se concluye en un paralelismo bastante estrecho para hierro sérico y $\%$ de saturación de la transferrina, y una menor velocidad de incremento de los valores de hematocrito y hemoglobina en los varones, to que viene a aparejarse alrededor de los 60 meses de edad. En todos los casos, los valores ob. servados para el sexo femenino a los 160 meses de edad, edad límite del estudio infantil, son inferiores a los del sexo masculino.

En relación a la concentración hemoglobínica

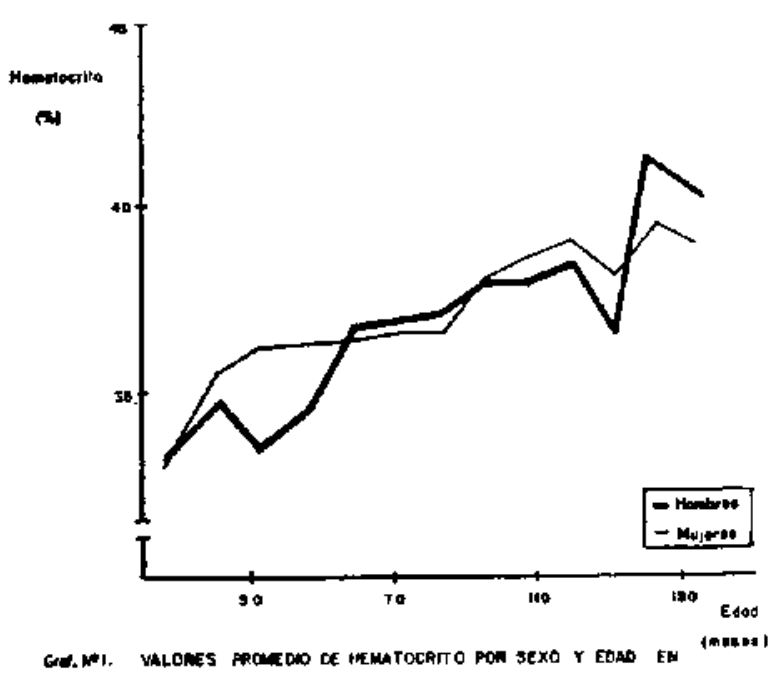

UNR POELACIOH MFANTIL DEL ANEA SUA OMENTE GE SANTL60

1970.

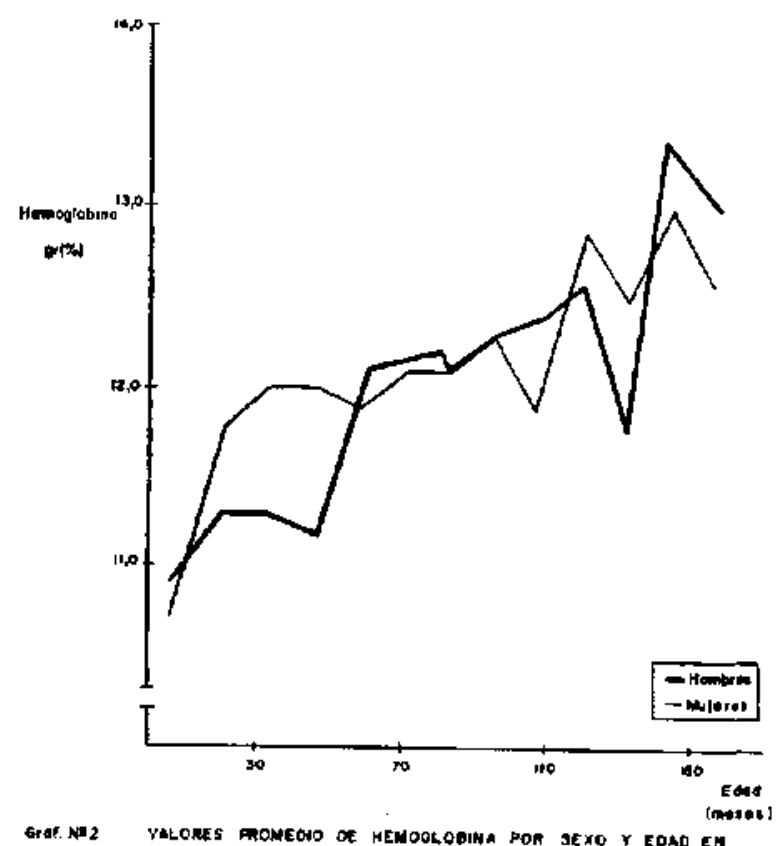

UNA POLLAGION WFANTL DEL AMEa SUA OAJENTE OE SAMTIAGO

1970.

corpuscular media, se observan valores promedio similares a través de la edad y en relación al sexo.

Los valores de transferrina muestran grandes fluctuaciones que dificultan su interpretación. EI becho más destacado es una reducción progresiva observada en forma paralela en ambos sexos desde los 20 hasta los 50 meses.

La distribución de los casos, en función de su suficiencia en relación a los patrones de referencia ya definidos, se representa en los gráficos N.os 7 al 11. 


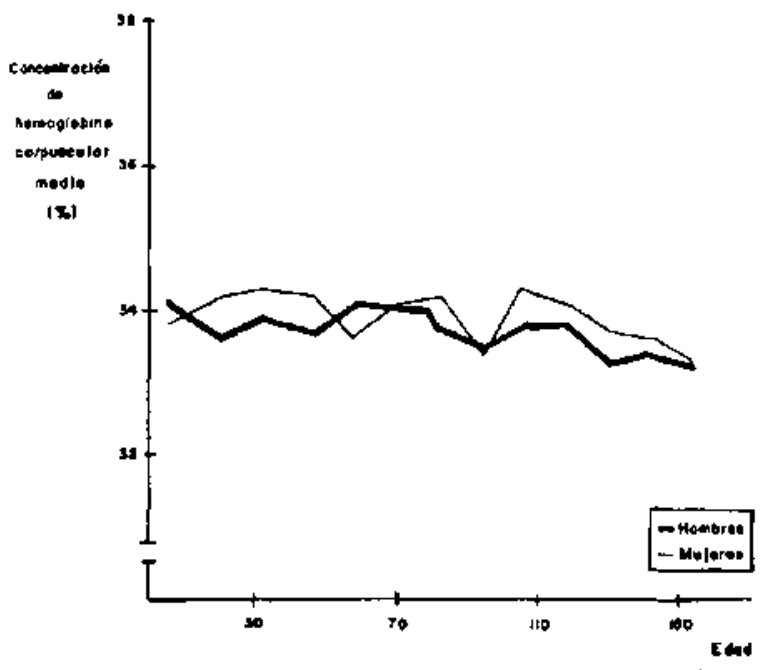

Arel Na3. VALOAES nOMEONO OE CONCENTRACION DE MEMOELOOWA

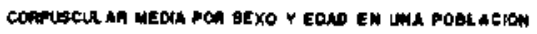

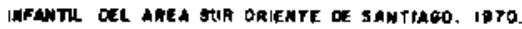

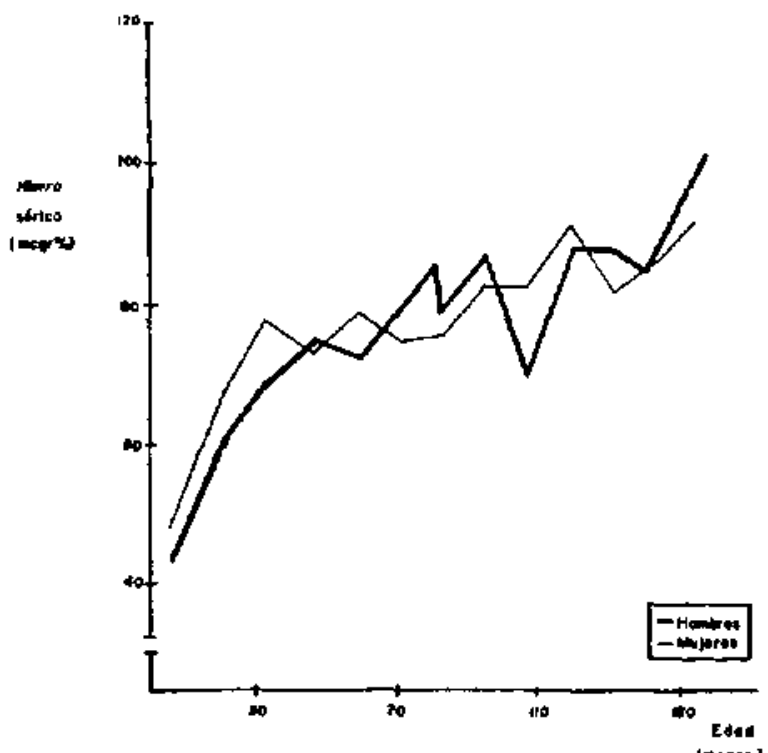

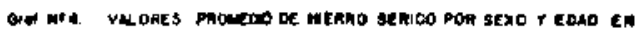

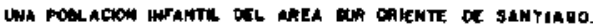

1970.

Un análisis global denota la existencia del más alto porcentaje de valores deficientes en la publación de lactantes, para disminuir paulatinamente en la edad preescolar y escolar, y subir nuevanicnte en la mujer a un nivel un poco superior al del preescolar. No se observa una diferencia significativa entre las condiciones de mujer nodriza y no nodriza. Analizando en cambio los indicadores en forma individual, el hierro sérico y el porcentaje de saturación de la transferrina, sobresalen como los más deficitarios, y la concentración hemoblobínica corpuscular media, como la menos afectada.

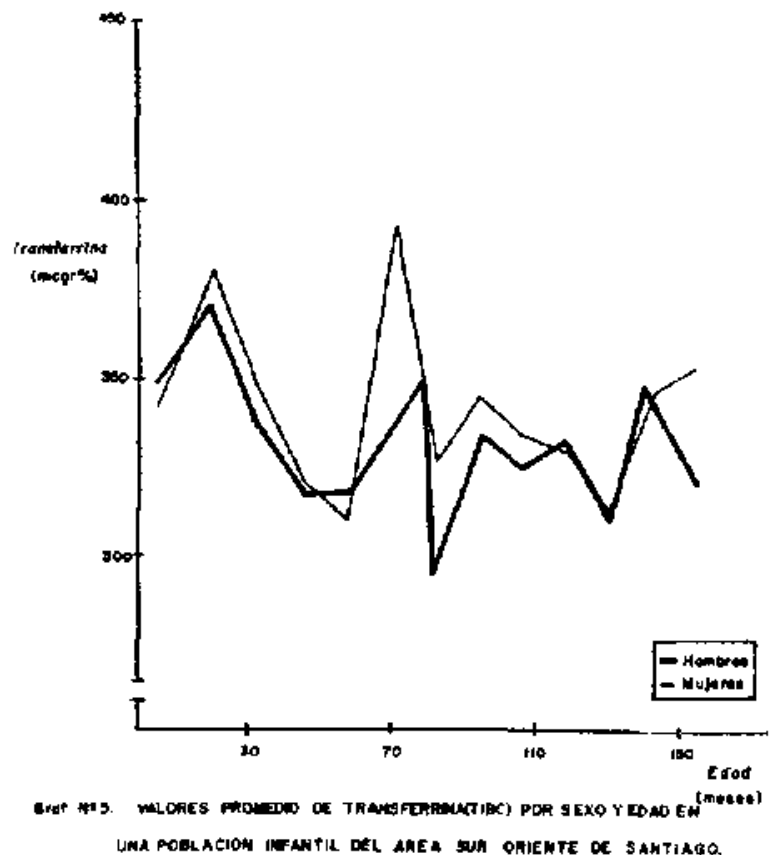

1970

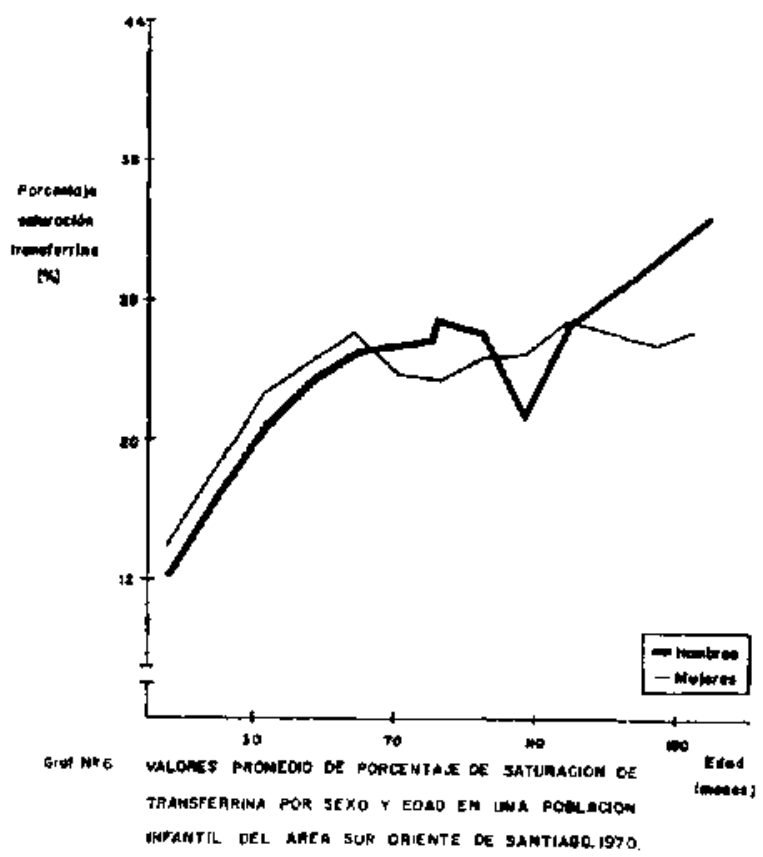

La tabia Nọ 5 muestra la preváéncia dis antmia ferropénica, de acuerdo a presentar un valor de hemoglobinemia $<10 \mathrm{gr} \%$ infancia $\mathrm{y}$ $<11$ gr\% mujeres y hierro sérico $<50 \mathrm{mcgr} \%$. Hay una prevalencia del $6,6 \%$ en la población dc lactantes, $2,4 \%$ en preescolares, $1,0 \%$ en escolares, $6,9 \%$ en mujeres no nodrizas y $7,5 \%$ en nodrizas.

La prevalencia de deficiencia de hierro apa- 


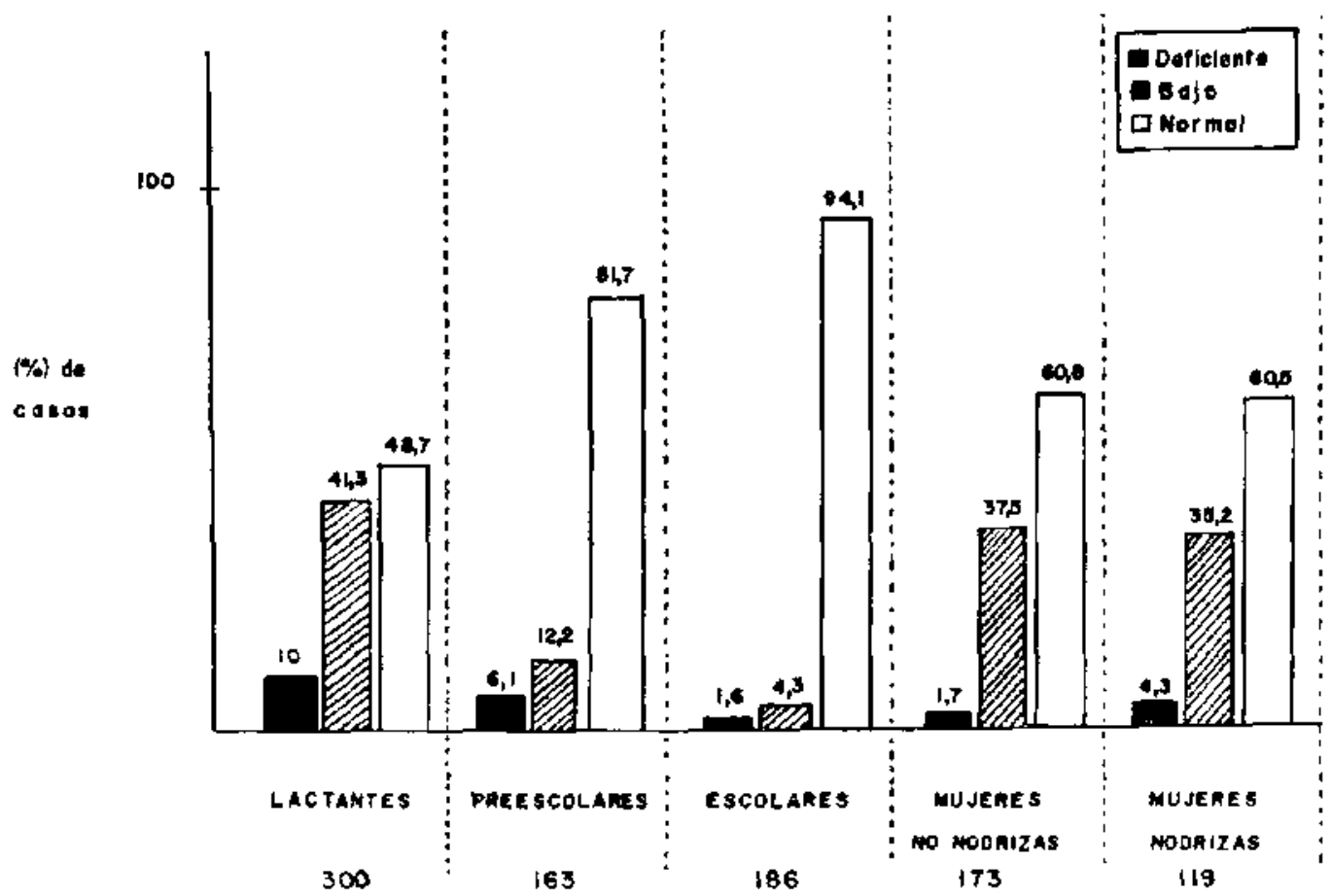

Grof N17. HEMATOCRITO. DISTRIBUCION DE CASOS SEGUN VALORES DE REFERENCIA poglacion materno infantil. ahea sur oriente de santiago.1970.

\section{T A B L A No 5}

APRECIACION DE LA PREVALENCIA DE ANEMIA FERROPENICA (') EN LA POBLACION MATERNOINFANTIL DEL AREA SUR ORIENTE DE SANTIAGO 1970

\begin{tabular}{l|c|c|c}
\hline \multicolumn{1}{c|}{ GRUPO } & $\begin{array}{c}N^{\circ} \text { casos } \\
\text { esthdiados }\end{array}$ & $\begin{array}{c}N^{\circ} \text { casos } \\
\text { con ane- } \\
\text { mia ferro- } \\
\text { priva }\end{array}$ & $\begin{array}{c}\% \text { casos } \\
\text { con } \\
\text { anemia } \\
\text { ferropriva }\end{array}$ \\
\hline Lactantes & 299 & 20 & 6,6 \\
Preescolares & 162 & 4 & 2,4 \\
Escolares & 187 & 2 & 1,0 \\
Mujeres nodrizas & 119 & 9 & 7,5 \\
Mujeres no nodrizas & 172 & 12 & 6,9 \\
\hline
\end{tabular}

$\left.{ }^{*}\right) \mathrm{Hb}<10 \mathrm{gr} \%$ en infancia.

$\mathrm{Hb}<11 \mathrm{gr} \%$ en adultos mujeres.

Hierro sérico $<50 \mathrm{mcgr} \%$.

rece en la tabla $N^{0} 6$, tomando un límite de $<15 \%$ de saturación de la transferrina. Destaca uni prevalencia del $75,9 \%$ para la población de lactantes, $25 \%$ para el preescolar, $21,6 \%$ para el escolar, $35,8 \%$ para la nodriza y $42,8 \%$ para la no nodriza.

En la tabla NQ 7 se aprecia el porcentaje de casos que a pesar de tener una deficiencia de hierro, presentaban valores normales de hematocrito, hemoglobinemia, concentración hemoglobínica corpuscular media y hierro sérico.

En todos los grupos fisiológicos cstudiados, hay un alto porcentaje: 48 a $91 \%$ presentaban hematocrito normal; entre $\$ 9,3$ y $95,4 \%$ concentración hemoglobinica corpuscular media normal; entre 74,4 y $96,0 \%$ heinoglobinenia normal y cntre 22,8 y $51,5 \%$ hierro sérico normal, siendo este último el ind:cador de mayor fidclidad relativa.

TA B LA No 6

APRECIACION DE LA DEFICIENCIA DE MIERRO EN LA POBLACION MATERNO-INFANTIL DEL AREA SUR ORIENTE DE SANTIAGO. 197O. (\% DE SATURACION DE TRANSFERRINA $<15 \%)$

\begin{tabular}{l|c|c|c}
\hline GRUPO & $\begin{array}{c}N^{\circ} \text { casos } \\
\text { estudiados }\end{array}$ & $\begin{array}{c}\text { No casos } \\
\text { con defi- } \\
\text { cienciat de } \\
\text { hierro }\end{array}$ & $\begin{array}{c}\text { so casos } \\
\text { con defi- } \\
\text { ciencia de } \\
\text { hierm }\end{array}$ \\
\hline Lictantes & 216 & 164 & 75,9 \\
Preescolares & 128 & 32 & 25,0 \\
Escolares & 171 & 37 & 21,6 \\
Mujares nodrizas & 106 & 38 & 35,8 \\
Mujeres no nodrizas & 161 & 69 & 42,8 \\
\hline
\end{tabular}




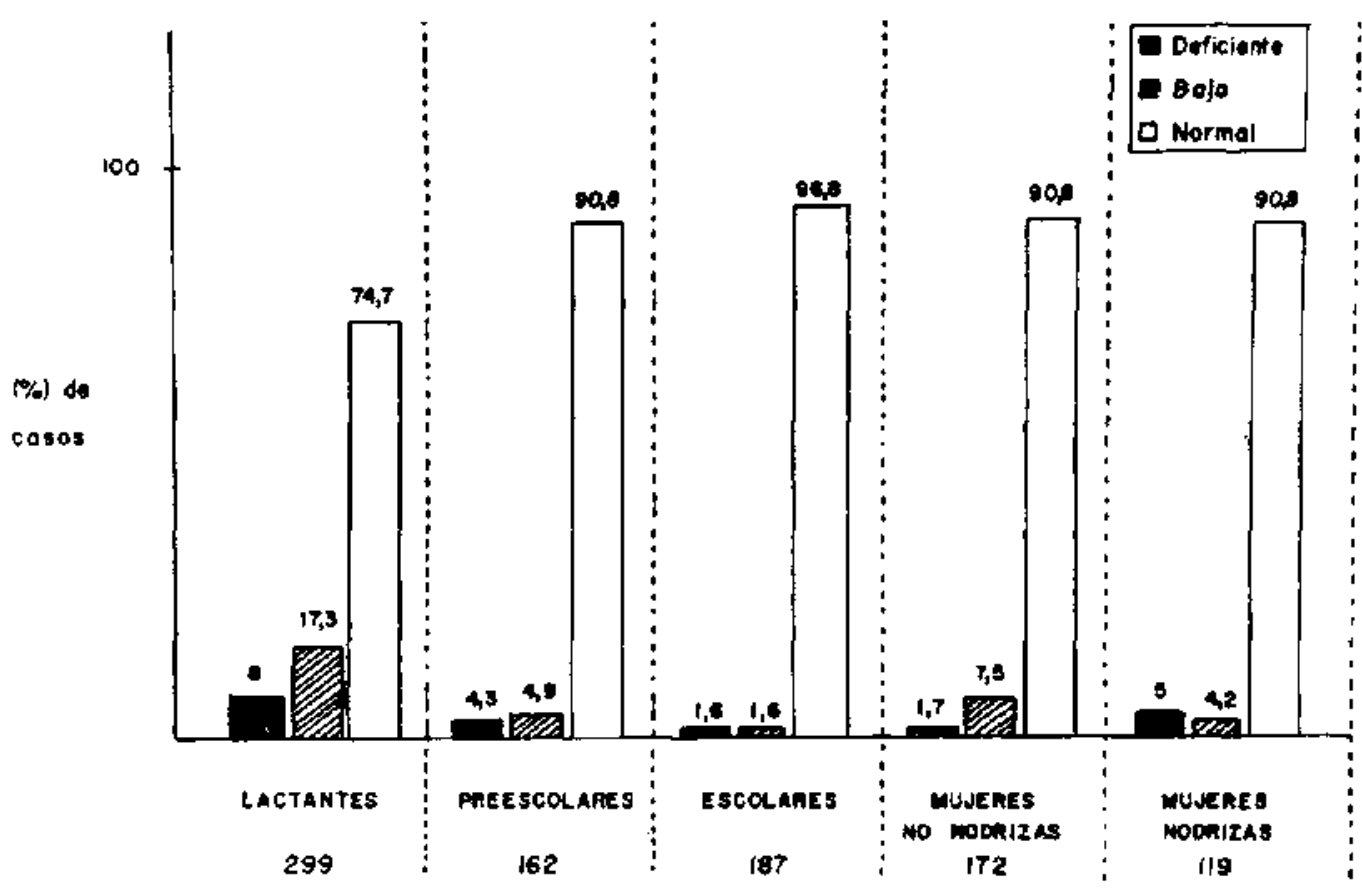

Grof. NRB. HEMOGLGBINEMIA. DISTRIBUCION DE CASOS SEGUN VALORES DE REFERENCIA. POALACION MATERHO-INFANTIL. AREA SUR ORIENTE DE SANTIAGO. 197O.

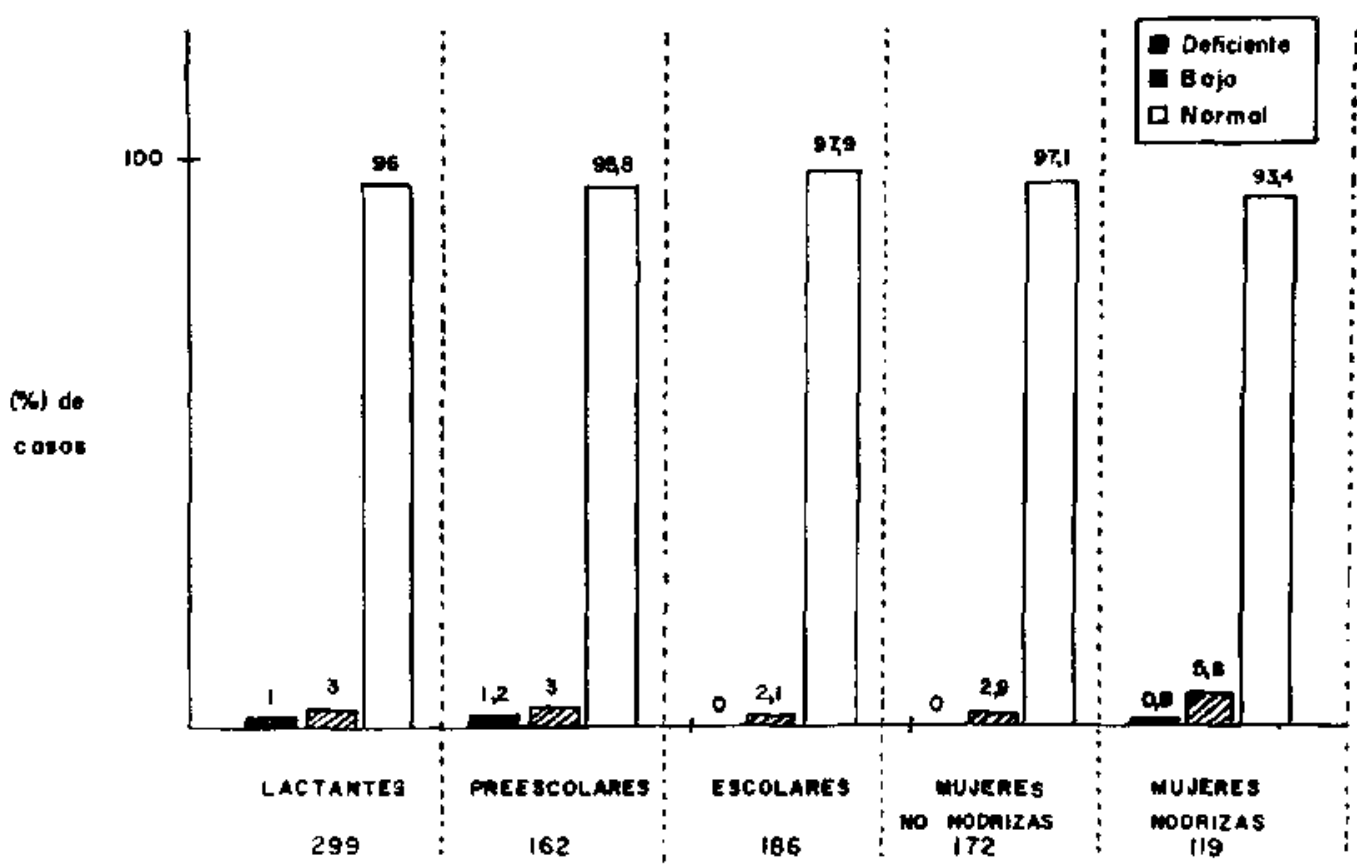

Grof. NE9. CONCENTRACION HEMOGLOBINICA CORPUSCULAR MEDIA. OISTRIGUCION DE CASOS SEGUN VALORES DE REFERENCIA. POglacion MATEANO-INFANTIL. AREA SUR ORIENTE DE SANTIAGO. 1970. 


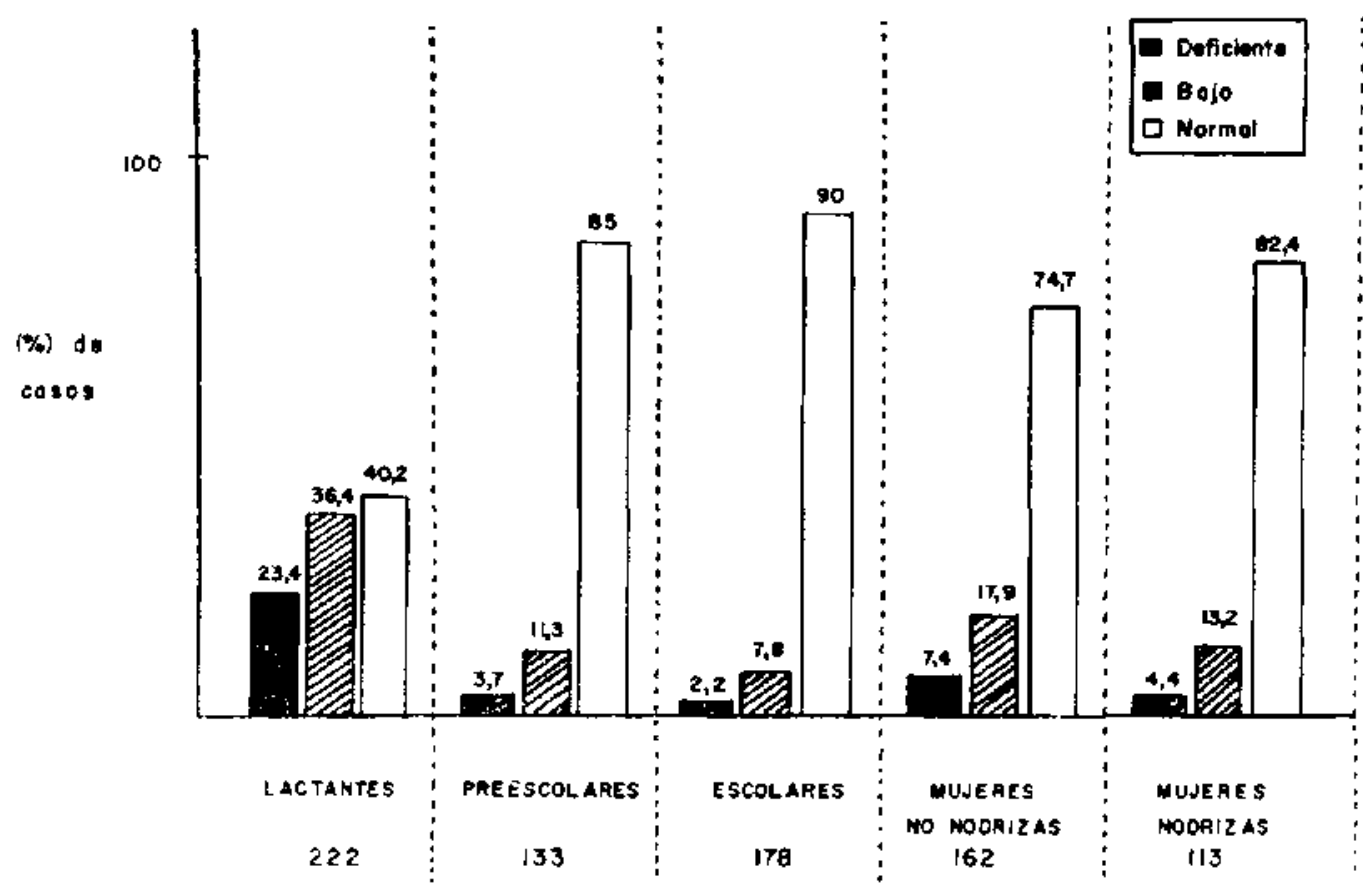

GrAf NE PO TERRO SERICO OISTRIBUCION DE CASOS SEGUN VALORES DE REFERENCIA. poblacion materno-infantil. area sur ORIENTE de santiago 1970.

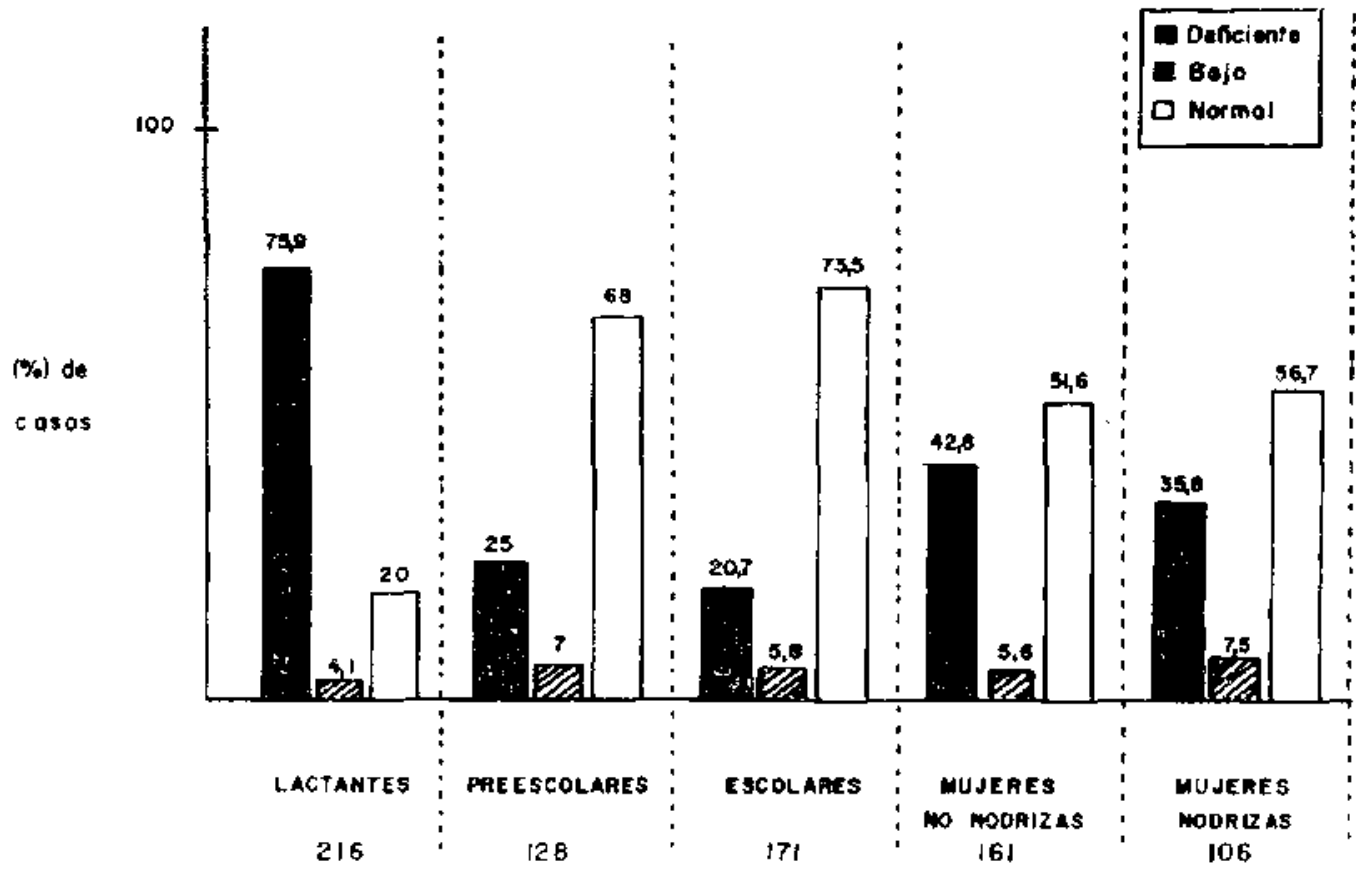

Grof N\$1 PORCENTAJE DE SATURACION DE TRANSFERRJAA, DISTRIBUCION DE CASCS SEGUN VALORES DE REFERENCIA. 
TAB L A No 7

APRECIACION DEL PORCENTAJE DE CASOS QUE, A PESAR DE TENER UNA DEFICIENCIA DE HIERRO $\%$ DE SATURACION DE TRANSFERRINA < I5\%), PRESENTABAN NIVEL DE HEMATOCRITO, HEMOGLOBINEMIA, CONCENTRACION HEMOGLOBINICA CORPUSCULAR MEDIA Y HIERRO SERICO, NORMAL. POBLACION MATERNO-INFANTIL. AREA SUR ORIENTE DE SANTIAGO, 1970

\begin{tabular}{|c|c|c|c|c|c|}
\hline Cosos de Ferropenia & $\begin{array}{l}\text { ractan- } \\
\text { tes } 164\end{array}$ & $\left\{\begin{array}{c}\text { Prees- } \\
\text { coldrex } \\
\text { res } 32\end{array}\right.$ & $\mid \begin{array}{l}\text { Gsconta- } \\
\text { res } 37\end{array}$ & $\begin{array}{c}\text { Afrife- } \\
\text { res ro- } \\
\text { frizas } \\
37\end{array}$ & $\mid \begin{array}{l}\text { Hete- } \\
\text { res no } \\
\text { jestri- } \\
\operatorname{2ex} 69\end{array}$ \\
\hline & $\%$ & $\%$ & $\%$ & $\%$ & 56 \\
\hline Hematocrito normal & 48,0 & | & 90,9 & 91.0 & $87, B$ \\
\hline Hóngglobincmia normal & 74,4 & ' 96,0 & 94,2 & 94,0 & 83.7 \\
\hline С. Н. C. M. лormal & i 94,0 & 93.4 & 91.0 & 95.4 & 89.3 \\
\hline Hietro sćrìco normal & | 22,8 & 38,5 & 51.5 & 43,6 & I 47,3 \\
\hline
\end{tabular}

Comentarios. Del presente trabajo se desprende que cl déficit de hierro y la anemia ferromiva constitiven un problema de alta prevalencia en la población infantil y materna del úrea en estudio. Ello adquiere su máxima expresión en la lactancia, en donde el $75.9 \%$ presenta un déficit de hierro y un 6,6\% anemia ferropriva. especialmente en el segundo semestre de vida. El problema decrece en magnitud en el preescolar y escolar, pero mantiene una prevalencia significativa: carencia de hierro en el $25.0 \%$ y $21,6 \%$, y anemia ferropriva en cl $2.4 \%$ y $1,0 \%$ respectivamente.

En la mujer adulta la prevalencia es mayor que en los escolares. alcanzando cifras de $35.8 \%$ y $42,8 \%$ de carencia de hierro y de $7.5 \%$ y $6,9 \%$ de anemia ferropriva en la mujer nodriza $y$ no nodriza respectivamente.

La explicación de la alta preva ${ }^{\dagger}$ encia del problema en la lactancia, obdece a la concurrencia de varios posibles factores: prematurez y desnutrición fetal, bajo aporte de hierro en la dieta, e infecciones entéricas y generales. La persistencia del problema en el preescolar y escolar se explica en parte por la grave depleción del lactante y también por un aporte de hierro dietético de bajo rendimiento absortivo, junto a un déficit calóricoproteico, como es posible observar en encuestas alimentarias a estos grupos (10). Es probable que la reducción paulatina de la prevalencia de la carencia de hierro, sea el reflejo de un incremento gradual de los depósitos de fierro, que colocan a los individuos en una situación de mayor seguridad frente a injurias.

La alta prevalencia en la mujer puede explicarse por el sangramiento menstrual, los frecuentes embarazos y períodos de lactancia. La ausencia de diferencias significativas en la prevalencia de anemia ferropriva entre la mujer nodriza y no nodriza, y la mejor situación de los depósitos de la primera, no nos permite apoyar la influencia de la lactancia en el deterioro de los depósitos de hierro.

En este estudio se pone cn relieve la insuficiencia de los indicadores ccmo ef hematocrito, hemo. globina sérica y concentración hemoglobiniza corpuscular media, para apreciar y cuantificar el problema de la carencia de hierro. No es nuestra intención invalidar dichos indicadores, que mantienen su vigencia en el diagnóstico de la anemia ferropénica, sino que señalar sus limitaciones en el análisis del problema. Bástenos señalar que la mujer no nodriza tiene una preva'encia de anemia ferropriva deì $6,9 \%$ y una carencia de hierro del $42.8 \%$ y el lactante una nrevalencia del $6.6 \%$ y una carencia de hierro del $75,9 \%$. Destacamos que la determinación de hierro sérico es la que da una mayor aproximación al criterio, internacionalmente accptado, de considerar como carencia de hierro a un bajo porcentaje de saturación de lat transferrina.

Una revisión de la literatura nacional a nuestro alcance, revela escasa información sobre la prevalencia de anemia ferropriva y carencia de hierro en Chile. Prácticamente no existen datos para preescolares, escolares y adultos.

Nosotros (4), encontramos un $25,9 \%$ de anemia en lactantes de 6 a 24 meses (hemoglobinemia $<10$ gx $\%$ ) y un $70,0 \%$ con hierro sérico menor de $40 \mathrm{mcgr} \%$. La población pertenecía a dos grupos sociales, incluyendo lactantes eutróficos y distróficos.

Margozzini y Cols. (5), en 292 lactantes eutróficos señala un $4.7 \%$ de anemia fêrroprivi (hemoglobinemia < $10 \mathrm{gr} \%$ ) y un $62.5 \%$ con saturación de transferrina menor del $15 \%$.

La alta prevalencia del problema en el áren estudiada, nos obliga a plantear la necesidad de planificar acciones para corregir este trastorno. Quizás las nás efectivas sean aquéllas dirigidas a repletar los depósitos de fjerco del lactante, ya sea mediante enriquecimiento de la leche, hierroterapia preventiva o cambios en las actitudes en la alimentación del lactante, incorporándolo precozmente a una alimentación no láctea, rica en bierro.

En el preescolar, escolar y mujer adulta, debe incorporarse un programa de detección y tratamiento, utilizando los controles del niño sano, de salud escolar y los programas de control materno.

Para terminar, crecmos imprescindible ampliar los estudios de prevalencia de este problema en la población chilena, estratificando con un criterio geogiáfico y de nivel social. Sólo así, con un conocimiento global del problema en el país, podremos planificar un programa a nivel nucional.

Pensamos que la anemia ferropénica constituye un importante factor de limitación de la población, menoscaba el rendimiento del preescolar 
y escolar y la capacidad física del aduito (16), to que constituye un nuevo obstáculo a muestras tentativas ae escapar del subdesarrollo humano y social.

RESUMEN y CONCLUSIONES. 1. Se determinan los niveles de hematocrito, hemoglobinemia, hierro sérico, transferrina y se calculan la concentración hemoglobinica corpuscular media y el $\%$ de saturación de la transferrina, en 404 lactantes entre 6 y 24 meses, 217 preescolares, 226 escolares, 189 mu:jeres no nodrizas y 136 nodrizas de la población del Area Sur Oriente de Santiago.

2. Se presentan los valores promedio para cada grupo y se analizan según edad y sexo en la pobJación infantil.

3. Se analiza la suficiencia de los valores individuales, comparándo os con patrones de normalidad internacional.

4. Se establece la prevalencia de anemia ferropriva (henoglobinemia $<10$ gr\% cn población infantil y $<11 \mathrm{gr} \%$ en mujeres y hierro sérico $<50$ megr $\%$ ). Lactantes $6,6 \%$, prescolares $2,4 \%$, escolares $1,0 \%$, mujeres nodrizas $7,5 \%$ y mujeres no nodrizas $6,9 \%$.

5. Se señala la prevacencia de carencia de hierro, utilizando como margen una saturación de transferriaa menor de $15 \%$ : lactantes $75,9 \%$, preescolares $25 \%$, escolarcs $21,6 \%$, mujeres nodrizas $35,8 \%$ y no nodrizas $42,8 \%$.

6. Se destacan las limitaciones del hematocrito, hemogiobinemia, concentración hemogiobínica corpuscular media y hierro sérico en el diagnóstico de la carencia de hicrro, señalando al hierro sérico como el más aproximado.

7. Se señala la necesidad de ampliar los estudios para obtener una visión nacional del problema y poder realizar una planificación adecuada de los programas correctivos.

8. Se plantea la necesidad de iniciar acciones en el área en estudio. Se plantea como acción prioritaria la corrección del problema del lactante, ya sea a través de enriquecimiento de la leche, hie. rroterapia preventiva o cambios en la técuica de alimentación. En preescolares, escolares y mujeres, una campaña de dctección y tratamiento masivo de estc trastorno.

\section{BiBliografía}

1.-Organización Mundial de la Salud. Grupo científico de la OMS sobre anemias nutricionales. Inf. Serv. Tec. Na 405, Ginebra, 1968.

2.-. Organización Mundial de la Salud. Nutrición. EI programa de la OMS. 1965-71, Ginebra. 1972.

3.-Daridson S. and Passmore R. "Humat Nutrition and Dietetis". Third Edit. E \& S. Livingstonc. Edinburgh \& London, 1966.
4.-Winter A., Taboada H., Mena F. ,Molina K. "Anemia hipocroma ferropriva en el lactante". Rev. Chilena de Pediatría. 32: 649, 1961.

5.-Margozzini J., Bravo M, Lanzkowsky P., Valiente S., Rosales E., Urteaga C., Vurgas $S_{+}$López L., Covarubias E., Pusatic O., Arauco C. "La cirencia de hierro en el lactante considerado eutrófico". Area Hospitalaria Norte de Santiago, Rev. Chilena de Pediatría. 48: 9, 1972.

6.- Zerteno T., Consa O., Grunewal $O$. "Recuento de glóbulos rojos y blancos, determinación de hematocrito, bemoglobina y volumen corpuscular medio en 893 lactantes y niños desde la primera semana de vida hasta los 7 años". Rev. Chilena de Padiatríd. 21: 99, 1950.

7.-Id. II Comunicación. Coeficiente de variación. Rev. Chilena de Pediatría. 21: 11 1, 1950.

8.- Id. II Comanicación. Estudio de la significación estadísticat de los promedios. Rev. Chilena da Pedistría. 21: 163, 1950.

9.- h. IV Coimunicación. Estudio estadistico del grildo de correlación entre los valores de hematocrito y volumen corpuscular medio en grupo de ni.ios menores dz I año a 7 anoos de edad. Rev. Chilena de Pediatría. 21: 219, 1950.

10.- Arteaga A., Winter A., Taboada H., Galufré A., Maiz A., Cornejo L. "Estudio de la interacción entre nutrición y anemia ferropriva en una población m.aterno-infantil del Area Sur Oriente de Suntiago". (Ex pransa).

11.- PAHO/WHO. "Standarizing methodology and Computer analysis for assesing Nutrition. Guids for interpretación of survey findigs". Buenos Aires. Argentina, 1971.

12.-Dacy J. V. and S. M. Lewis. "Haemoglobin (as cyanmetha-haemoglobin ) Practical haematology. pg. 37 Grune \& Stratton 3 Edith. N. Y. 1963.

13.- Mc. Govem J., Richarson A., Stcinberg A. "The hematocrit of capillary blood" New England $J$. of Med. 253: 308, 1955.

14.-Bothmell T. H. and Malletf B. "The determination of iron in plasma or serum" Biochem. J. 59: 599, 1955.

15.- Infermational Comitec for Standarization in hacmarology. Brit. J. of Haematology $20: 451,1971$.

16.- Arteuga A., Donuso H., Apud E., Rosales E. "Re. lación entre estado nutritivo y capacidad f́́sica. Estudio realizado en obreros chilenos". Rev. Med. de Chile. 99: 904, 1971.

Trahajo financiado con fondos LSAID proyecto 513-11-5\%1-243.

\section{AGRADECIMIENTOS}

Nuestrus agradecimientos a los Ingenicros Eduat do Varas y Bonifacio Fctnindez, por su participación sol la programación y compunación de los resultados 